\title{
Erratum: Gravitational Waves from Quasicircular Black-Hole Binaries in Dynamical Chern-Simons Gravity [Phys. Rev. Lett. 109, 251105 (2012)]
}

Kent Yagi®, Nicolás Yunes, and Takahiro Tanaka

(Q) (Received 2 January 2020; published 16 January 2020)

DOI: 10.1103/PhysRevLett.124.029901

We found some typos in Eq. (5) in the Erratum to this Letter, which is now corrected to

$$
\delta C_{\mathcal{F}} \equiv \frac{25}{24576} \zeta \frac{1}{\eta^{2}}\left[\Delta^{2}+27\left\langle\left(\Delta \cdot \hat{v}_{12}\right)^{2}\right\rangle_{\omega}\right]
$$

This modifies Eq. (6) of the Erratum to

$$
\begin{aligned}
\delta C= & \frac{101555}{344064} \zeta \frac{m^{2}}{m_{1}^{2}} \chi_{1}^{2}\left[1-\frac{58833}{20311}\left(\hat{\boldsymbol{S}}_{1} \cdot \hat{\boldsymbol{L}}\right)^{2}\right] \\
& -\frac{12725}{49152} \zeta \frac{\chi_{1} \chi_{2}}{\eta}\left[\left(\hat{\boldsymbol{S}}_{1} \cdot \hat{\boldsymbol{S}}_{2}\right)-\frac{1467}{509}\left(\hat{\boldsymbol{S}}_{1} \cdot \hat{\boldsymbol{L}}\right)\left(\hat{\boldsymbol{S}}_{2} \cdot \hat{\boldsymbol{L}}\right)\right]+(1 \leftrightarrow 2) .
\end{aligned}
$$

The parametrized post-Einsteinian parameter $\beta_{\mathrm{ppE}}$ computed from Eq. (2) correctly reduces to Eq. (3) of Ref. [1] in the spinaligned case and keeping only to quadratic order in spin. We have checked that the correction to $\beta_{\mathrm{ppE}}$ is very small and does not give any noticeable difference to our final result in Fig. 1 in the Erratum to this Letter.

[1] R. Nair, S. Perkins, H. O. Silva, and N. Yunes, Phys. Rev. Lett. 123, 191101 (2019). 